\title{
SYMMETRIES OF SPHERICAL HARMONICS $\left({ }^{1}\right)$
}

\author{
BY \\ ROBERTO DE MARIA NUNES MENDES
}

\begin{abstract}
Let $G$ be a group of linear transformations of $R^{n}$ and $H_{k}(G)$ the vector space of spherical harmonics invariant under $G$. The Pólya function is the formal power series $\Sigma_{k \geqslant 0} t^{k} \operatorname{dim} H_{k}(G)$. In this paper, after classifying all closed subgroups of $O(4)$, we compute the Poblya functions for these groups. These functions have recently proved to be of interest in quantum mechanics and elementary particle physics.
\end{abstract}

1. Pólya functions. We denote by $S^{n-1}$ the unit sphere in euclidean space $R^{n}$, by $O(n)$ the orthogonal group of $R^{n}$, by $S O(n)$ the subgroup of $O(n)$ consisting of those $\sigma \in O(n)$ such that $\operatorname{det} \sigma=1 . G$ denotes a fixed closed subgroup of $O(n)$.

Definition. A spherical harmonic $f_{k}$ of degree $k$ is the restriction to $S^{n-1}$ of a homogeneous polynomial $F_{k}(x)$ of degree $k$ which is harmonic.

We have $x=\left(x_{1}, \cdots, x_{n}\right) \in R^{n},|x|=\left(x_{1}^{2}+\cdots+x_{n}^{2}\right)^{1 / 2}$ and $F_{k}(x)$ $=r^{k} f_{k}(x / r)$ for $r=|x| \neq 0$.

We denote by $H_{k}$ the vector space of spherical harmonics of degree $k$, and by $H_{k}(G)$ the vector subspace of those invariant under $G$. The Polya function is defined by $F_{G}(t)=\Sigma_{k \geq 0} t^{k} \operatorname{dim} H_{k}(G)$. The classical Molien formula is

$$
F_{G}(t)=\int_{G} \frac{1-t^{2}}{\operatorname{det}(1-t \sigma)} d \sigma
$$

where $d \sigma$ is the normalized Haar measure on the group $G$. If $G$ is a closed subgroup of $S O(4)$, we have

$$
F_{G}(t)=\int_{G} \frac{1-t^{2}}{\left(1-2 t \cos \alpha_{\sigma}+t^{2}\right)\left(1-2 t \cos \beta_{\sigma}+t^{2}\right)} d \sigma
$$

where $\alpha_{\sigma}, \beta_{\sigma}$ are the angles of the rotation $\sigma$.

Received by the editors September 18, 1973.

AMS (MOS) subject classifications (1970). Primary 22E30, 22 C05.

Key words and phrases. Orthogonal group, generating function, spherical harmonic, closed subgroup, invariant.

(1) This paper is based on the author's doctoral dissertation written at University of California at San Diego under the supervision of Professor J. P. Fillmore, to whom he is most grateful. The author was partly supported by Conselho Nacional de Pesquisas and Universidade Federal de Minas Gerais, Brasil. 
REMARK. Pólya and Meyer computed the generating function $F_{G}(t)$ for the finite subgroups of $O(3)$. Their results are given in [11].

We want to compute the function $F_{G}(t)$ for the closed subgroups of $O(4)$. Then our first task is to determine these subgroups.

2. Subgroups of $S O(4)$. Any rotation in $R^{4}$ can be written as a mapping $q \rightarrow l q r^{-1}$ where $q=(w, x, y, z)=w+x i+y j+z k$ is a quaternion and $l, r$ are unit quaternions. Let $\varphi: S^{3} \times S^{3} \rightarrow S O(4)$ be the 2-1 homomorphism defined by $\varphi(l, r)(q)=l q r^{-1}$. Let $G \subset S O(4)$ be a closed subgroup and $A=$ $\varphi^{-1}(G)$. Let $\mathrm{pr}_{i}: S^{3} \times S^{3} \rightarrow S^{3}, i=1,2$, denote the projection onto the $i$ th factor. Set $L=\operatorname{pr}_{1} A, R=\operatorname{pr}_{2} A ; L$ and $R$ are closed subgroups of $S^{3}$. Set $L_{K}=\{l \in L ;(l, 1) \in A\}, R_{K}=\{r \in R ;(1, r) \in A\} ; L_{K}$ (resp. $R_{K}$ ) is a closed normal subgroup of $L$ (resp. $R$ ). We have an isomorphism of Lie groups, $\Phi$ : $L / L_{K} \rightarrow R / R_{K}$, given by $\Phi\left(l L_{K}\right)=r R_{K}$, where $(l, r) \in A$.

Conversely, given $L, R, L_{K}, R_{K}, \Phi$ as above we define the group $G$ by $G=\left\{g \in S O(4) ; g(q)=l q r^{-1}\right.$ with $\left.l \in L, r \in R, \Phi\left(l L_{K}\right)=r R_{K}\right\}$.

If $A=\varphi^{-1}(G)$ then $\operatorname{pr}_{1} A=L, \operatorname{pr}_{2} A=R,\{l \in L ;(l, 1) \in A\}=L_{K}$, $\{r \in R ;(1, r) \in A\}=R_{K}$. We use the notation $G=\left(L / L_{K} ; R / R_{K}\right)_{\Phi}$ or $G=\left(L / L_{K} ; R / R_{K}\right)$ when $\Phi$ is clearly understood.

Proposition. Let $G=\left(L / L_{K} ; R / R_{K}\right)_{\Phi}$ and $H=\left(M / M_{K} ; N / N_{K}\right)_{\Psi}$ be two subgroups of $S O(4)$. Then

(i) there exists $\gamma \in S O$ (4) such that $G=\gamma H_{\gamma}^{-1}$ if and only if there exist isomorphisms of Lie groups $\alpha: L / L_{K} \rightarrow M / M_{K}, \beta: R / R_{K} \rightarrow N / N_{K}$ of the form $\alpha\left(l L_{K}\right)=a^{-1} l a M_{K}, \beta\left(r R_{K}\right)=b^{-1} r b N_{K}$, where $a, b \in S^{3}$ and $\Psi \alpha=\beta \Phi$. Moreover, $a$ and $b$ are such that $L=a M a^{-1}, R=b N b^{-1}$;

(ii) there exists $\gamma \in O(4)-S O(4)$ such that $G=\gamma \mathrm{H \gamma}^{-1}$ if and only if there exist isomorphisms $\alpha: N / N_{K} \rightarrow L / L_{K}, \beta: R / R_{K} \rightarrow M / M_{K}$ of the form $\alpha\left(n N_{K}\right)=a n a^{-1} L_{K}, \beta\left(r R_{K}\right)=b^{-1} r b M_{K}$ where $a, b \in S^{3}$ and $\beta \Phi \alpha \Psi=1$. Moreover, $a$ and $b$ are such that $L=a N a^{-1}, R=b M b^{-1}$.

Proof. (i) If $\gamma(q)=a q b^{-1}$ is such that $G=\gamma H \gamma^{-1}$, then the elements of $G$ are of the form $g(q)=\left(a m a^{-1}\right) q\left(b n b^{-1}\right)^{-1}$ where $m \in M, n \in N$, $\left(m M_{K}\right)=n N_{K}$. Then $L=a M a^{-1}, R=b N b^{-1}$. Define $\alpha\left(l L_{K}\right)=a^{-1} l a M_{K}$, $\beta\left(r R_{K}\right)=b^{-1} r b N_{K}$. Then $\alpha, \beta$ are isomorphisms and $\Phi\left(a m a^{-1} L_{K}\right)=b n b^{-1} R_{K}$ implies that $\Psi \alpha=\beta \Phi$.

Conversely, if there exist isomorphisms $\alpha, \beta$ as above then define $\gamma(q)=$ $a q b^{-1}$. It is easy to see that $G=\gamma H \gamma^{-1}$.

The proof of (ii) is similar. 
Subgroups of $S^{3}$. The proper closed subgroups of $S O(3)$ are (up to conjugacy):

$T=$ tetrahedral group of order 12 ;

$0=$ octahedral group of order 24 ;

$I=$ icosahedral group of order 60 ;

$C_{n}=$ cyclic group of order $n$;

$D_{n}=$ dihedral group of order $2 n$;

$H_{1}=$ group of all rotations about a given fixed axis;

$\mathrm{H}_{2}=$ group of all rotations about a given fixed axis together with rotations of $\pi$ about all axes perpendicular to the given axis.

We have the 2-1 homomorphism $\pi: S^{3} \rightarrow S O(3)$ given by $\pi(p)(q)=$ $p q p^{-1}$. Then we obtain all the closed subgroups of $S^{3}$ taking $G=\pi^{-1}(G)$, where $G$ is a closed subgroup of $S O(3)$. We obtain the following proper subgroups of $S^{3}$ :

$T=\pi^{-1}(T)$ of order 24

$O=\pi^{-1}(O)$ of order 48 ;

$I=\pi^{-1}(I)$ of order 120 ;

$C_{n}=\pi^{-1}\left(C_{n}\right)$ if $n$ is odd; $\pi^{-1}\left(C_{n / 2}\right)$ if $n$ is even, of order $n$;

$D_{n}=\pi^{-1}\left(D_{n}\right)$ of order $4 n$;

$H_{1}=\pi^{-1}\left(H_{1}\right) . H_{1}$ is connected and $H_{1}=(\cos \theta, 0,0, \sin \theta) ; 0 \leqslant \theta \leqslant 2 \pi$,

$H_{2}=\pi^{-1}\left(H_{2}\right) . H_{2}=H_{1} \cup H_{1} i$.

With these subgroups we can now determine all the closed subgroups of $S O(4)$. The complete list appears in the table at the end of the paper. For the finite subgroups of $S O(4)$ the list is given in [7].

REMARK. There is only one group (up to conjugacy) of the form $\left(S^{3} / L_{K} ; S^{3} / R_{K}\right)_{\Phi},\left(H_{1} / C_{n} ; H_{1} / C_{n}\right)_{\Phi},\left(H_{2} / C_{n} ; H_{2} / C_{n}\right)_{\Phi}$.

3. Subgroups of $O(4)$. If $q=(w, x, y, z)$ then $\bar{q}=(w,-x,-y,-z)$ is the conjugate of $q$. The mapping $q \rightarrow \bar{q}$ is a reflection in $R^{4}$, and the resultant of this reflection with a general rotation $q-l q r^{-1}$ is the reflection $q \rightarrow$ $l \vec{q} r^{-1}$, and any reflection can be put in this form, i.e., the general reflection in $R^{4}$ has the form $q \rightarrow a \bar{q} b$ with $|a|=|b|=1$. If $B(q)=a \bar{q} b$ and $g(q)=$ $l q r^{-1}$ then $B g B^{-1}(q)=\left(a r a^{-1}\right) q\left(b^{-1} l b\right)^{-1}$.

Let $G^{*}$ be a subgroup of $O(4)$ which is closed and has $G=\left(L / L_{K} ; R / R_{K}\right)_{\Phi}$ as its pure subgroup. If $B \in G^{*}-G, B(q)=a \bar{q} b$, then the mapping $g \in G \rightarrow B g B^{-1} \in G$ is an automorphism of $G$, and we have $L=a R a^{-1}$, $L_{K}=a R_{K} a^{-1}, R=b^{-1} L b, R_{K}=b^{-1} L_{K} b$, that is, $L$ and $R$ are conjugate in $S^{3}$, and so are $L_{K}$ and $R_{K}$. It is easy to see that there exists an automorphism $\Psi: L / L_{K} \rightarrow L / L_{K}$ such that $G$ and $H=\left(L / L_{K} ; R / R_{K}\right)_{\Psi}$ are conjugate. Since 
we are interested in $G^{*}$ only up to conjugacy, it follows that we can take $L=R$ and $L_{K}=R_{K}$, and we have $L=a L a^{-1}=b L b^{-1}, L_{K}=a L_{K} a^{-1}=b L_{K} b^{-1}$, showing that $a$ and $b$ must belong to the normalizers of $L$ and $L_{K}$ in $S^{3}$. If $B \in G^{*}, B(q)=a \bar{q} b$, then its square is $q \rightarrow\left(a b^{-1}\right) q\left(b^{-1} a\right)$ and belongs to $G$, whence $a b^{-1} \in L$, which shows that $a, b$ belong to the same coset of $L$ in the intersection of the normalizers of $L$ and $L_{K}$ in $S^{3}$.

If $G^{*}$ has $G=\left(L / L_{K} ; L / L_{K}\right)_{\Phi}$ as its pure subgroup then define automorphisms $\alpha\left(l L_{K}\right)=a l a^{-1} L_{K}, \beta\left(l L_{K}\right)=b l b^{-1} L_{K}$, where $B(q)=a \bar{q} b$ and $B \in G^{*}$; if $\Phi\left(L_{K}\right)=r L_{K}$ then $\Phi\left(a r a^{-1} L_{K}\right)=b^{-1} l b L_{K}$ and $\beta \Phi \alpha \Phi=1$.

Accordingly we have the following cases of subgroups of $O(4)$, not in $S O(4)$, with dimension greater than zero:

(a) $G=\left(S^{3} / S^{3} ; S^{3} / S^{3}\right)=S O(4)$. We have $G^{*}=O(4)$. The reflections are $q \rightarrow a \bar{q} b$ with $a, b \in S^{3}$.

(b) $G=\left(S^{3} / C_{2} ; S^{3} / C_{2}\right)$. The reflections of $G^{*}$ are $q \rightarrow a \bar{q} b$ with $a, b \in S^{3}$ and $a b^{-1}= \pm 1$.

(c) $G=\left(S^{3} / C_{1} ; S^{3} / C_{1}\right)$. We have two subgroups $G_{1}^{*}=G \cup g_{1}^{*} G$ and $G_{2}^{*}=G \cup g_{2}^{*} G$ containing $G$ as pure subgroup, where $g_{1}^{*}(q)=\bar{q}$ and $g_{2}^{*}(q)=-\bar{q} . G_{1}^{*}, G_{2}^{*}$ are not conjugate.

(d) $G=\left(\mathrm{H}_{2} / \mathrm{H}_{2} ; \mathrm{H}_{2} / \mathrm{H}_{2}\right)$. The reflections of $G^{*}$ are $q \rightarrow a \bar{q} b$ with $a, b \in H_{2}$.

(e) $G=\left(H_{2} / H_{1} ; H_{2} / H_{1}\right)$. We have two groups, $G_{1}^{*}$ and $G_{2}^{*}$, having $G$ as pure subgroup, the reflections of $G_{1}^{*}$ (resp. $G_{2}^{*}$ ) are $q \rightarrow a \bar{q} b$ with $a, b \in H_{2}$, $a b \in H_{1}$ (resp. $q \rightarrow a \bar{q} b$ with $a, b \in H_{1} i$ ).

(f) $G=\left(H_{1} / H_{1} ; H_{1} / H_{1}\right)$. The reflections of $G^{*}$ are $q \rightarrow a \bar{q} b$ with $a, b \in H_{1}$.

(g) $G=\left(H_{1} / C_{n} ; H_{1} / C_{n}\right)$. We have three groups having $G$ as pure subgroups:

$G_{1}^{*}$-with reflections $q \rightarrow l \bar{q} r^{-1}$;

$G_{2}^{*}$-with reflections $q \rightarrow l \rho^{1 / 2} i^{-1} \bar{q} i r^{-1}$;

$G_{3}^{*}$-with reflections $q \rightarrow l \rho i^{-1} \bar{q} i r^{-1}$, where $l, r \in H_{1}, l r^{-1} \in C_{n}$ and $\rho^{1 / 2}=(\cos -\pi / n-, 0,0, \sin -\pi / n)$.

(h) $G=\left(H_{2} / C_{n} ; H_{2} / C_{n}\right)$. We have two groups, $G_{1}^{*}$ and $G_{2}^{*}$, having $G$ as pure subgroup. The reflections of $G_{1}^{*}$ (resp. $G_{2}^{*}$ ) are of the form $q \rightarrow$ $l \rho^{1 / 2} \bar{q} r^{-1}$ (resp. $q \rightarrow l \rho \bar{q} r^{-1}$ ) where $l, r \in H_{2}$ and $l r^{-1} \in C_{n}$.

4. Examples of computation of the Pólya function. If $G$ is a closed subgroup of $S O(4)$ then we have

$$
F_{G}(t)=\int_{A} \frac{1-t^{2}}{\left(1-2 t \cos [\alpha(a)-\beta(a)]+t^{2}\right)\left(1-2 t \cos [\alpha(a)+\beta(a)]+t^{2}\right)} d a,
$$


where $l=(\cos \alpha, b \sin \alpha, c \sin \alpha, d \sin \alpha), r=(\cos \beta, m \sin \beta, n \sin \beta, p \sin \beta)$, $b^{2}+c^{2}+d^{2}=m^{2}+n^{2}+p^{2}=1$ and $g=\varphi(l, r), \alpha$ and $\beta$ being functions of $a \in A$, and $\int_{A} d a=1$.

EXAMPLE 1. $G=\left(H_{1} / H_{1} ; H_{1} / H_{1}\right) . G$ is a connected subgroup of $S O(4)$ of dimension two. Here, $A=\varphi^{-1}(G)=H_{1} \times H_{1}$ and $d a=\left(1 / 4 \pi^{2}\right) d \alpha d \beta$. Then,

$$
F_{G}(t)=\frac{1-t^{2}}{4 \pi^{2}} \int_{0}^{2 \pi} \int_{0}^{2 \pi} \frac{d \alpha d \beta}{\left(1-2 t \cos (\alpha-\beta)+t^{2}\right)\left(1-2 t \cos (\alpha+\beta)+t^{2}\right)}=\frac{1}{1-t^{2}} .
$$

In order to treat the next example we need the lemma that follows. I am grateful to Professor T. Ganelius for showing me the technique used in its proof.

LEMMA.

$$
\sum_{k=0}^{n-1} \frac{1}{1-2 t \cos (2 k / n) \pi+t^{2}}=n \frac{1+t^{n}}{\left(1-t^{2}\right)\left(1-t^{n}\right)} .
$$

Proof. The sum of all the residues (in the extended plane) of a rational function is zero. We get the result considering the function

$$
f(z)=n z^{n} /(z-t)(1-t z)\left(z^{n}-1\right) .
$$

EXAMPLE 2. $G=\left(H_{1} / H_{1} ; C_{2 n} / C_{2 n}\right) . G$ has dimension one and $n$ components. We have $A=H_{1} \times C_{2 n}, A_{0}=H_{1} \times\{1\}$, where $A_{0}$ is the component of the identity of $A$. Then,

$$
F_{G}(t)=\frac{1}{2 n} \int_{A_{0}} F(\varphi(l, 1)) d a_{0}+\cdots+\frac{1}{2 n} \int_{A_{0}} F\left(\varphi\left(l, \rho^{2 n-1}\right)\right) d a_{0},
$$

where $l=(\cos \alpha, 0,0, \sin \alpha) \in H_{1}, \rho=(\cos (\pi / n), 0,0, \sin (\pi / n))$ and $d a_{0}=$ $d \alpha / 2 \pi$. Now, $\varphi\left(l, \rho^{k}\right)=$ rotation of angles $(\alpha-k \pi / n),(\alpha+k \pi / n)$. Therefore,

$$
\begin{aligned}
F_{G}(t) & =\frac{1}{2 n} \sum_{k=0}^{2 n-1} \frac{1}{2 \pi} \int_{0}^{2 \pi} \frac{1-t^{2}}{\left[1-2 t \cos (\alpha-k \pi / n)+t^{2}\right]\left[1-2 t \cos (\alpha+k \pi / n)+t^{2}\right]} d \alpha \\
& =\frac{1}{2 n} \sum_{k=0}^{2 n-1} \frac{1+t^{2}}{1-2 t^{2} \cos (2 k \pi / n)+t^{4}}=\frac{1+t^{2 n}}{\left(1-t^{2}\right)\left(1-t^{2 n}\right)} .
\end{aligned}
$$

EXAmple 3. $G=(T / V ; T / V)$. $G$ has 96 elements.

We have $T=V \cup V_{1} \cup V_{2}$, where $V=\{ \pm 1, \pm i, \pm j, \pm k\}, V_{1}=t V$, $V_{2}=t^{2} V, t=1 / 2(1,1,1,1)$. The rotations of $G$ are schematically $V q V, V_{1} q V_{2}$, 
$V_{2} q V_{1}$. If $\theta_{1}, \theta_{2}$ are the angles of $q \rightarrow l q r^{-1}$ then we have the following table for the elements of $T$ :

$\begin{array}{ccr}\cos \theta_{1} & \cos \theta_{2} & \# \\ 1 & 1 & 1 \\ 0 & 0 & 12 \\ -1 & -1 & 1 \\ 1 & -1 & 18 \\ 1 / 2 & -1 & 32 \\ 1 & -1 / 2 & 32\end{array}$

We have

$$
96 F_{G}(t)=\sum_{G} \frac{1-t^{2}}{\left(1-2 t \cos \theta_{1}+t^{2}\right)\left(1-2 t \cos \theta_{2}+t^{2}\right)}
$$

and we get $F_{G}(t)=\left(1+t^{12}\right) /\left(1-t^{4}\right)^{2}\left(1-t^{6}\right)$.

ExAMPLE 4. $G^{*}=(T / V ; T / V)^{*}$. The reflections are of the form $q \rightarrow a \bar{q} b$ with $a, b$ both in $V$ or one each in $V_{1}, V_{2}$. If the angle between $a$ and $b$ is $\theta$ then $g^{*}: q \rightarrow a \bar{q} b$ is conjugate to $h^{*}: q \rightarrow p \bar{q} p^{-1}$ where $p=(\cos (\theta / 2)$, $0,0, \sin (\theta / 2))$. Then, $\operatorname{det}\left(1-t g^{*}\right)=\operatorname{det}\left(1-t h^{*}\right)=\left(1-t^{2}\right)\left(1+2 t \cos \theta+t^{2}\right)$ and we have

$$
F_{G^{*}}(t)=\frac{1}{2} F_{G}(t)+\frac{1}{2|G|} \sum_{G^{*}-G} \frac{1}{\left(1+2 t \cos \theta+t^{2}\right)}
$$

In our case we have the following values for $\cos \theta$ :

$\begin{array}{rr}\cos \theta & \# \\ 1 & 4 \\ -1 & 4 \\ 0 & 24 \\ 1 / 2 & 32 \\ -1 / 2 & 32\end{array}$

Therefore,

$$
F_{G} *(t)=\frac{1+t^{12}}{\left(1-t^{4}\right)\left(1-t^{6}\right)\left(1-t^{8}\right)}
$$

Example 5. $G=\left(S^{3} / C_{2} ; S^{3} / C_{2}\right) \cong O(3) . G$ has dimension 3 and 2 components. Here, $A=A_{0} \cup A_{1}$ where $A_{0}=\left\{(l, l) ; l \in S^{3}\right\}, A_{1}=\{(l,-l)$; 
$\left.l \in S^{3}\right\}$. An element of $S^{3}$ is given by $l=\left(\cos \theta_{1}, \sin \theta_{1} \cos \theta_{2}, \sin \theta_{1} \sin \theta_{2} \cdot\right.$ $\left.\sin \theta_{3}, \sin \theta_{1} \sin \theta_{2} \cos \theta_{3}\right)$ and the volume element of $S^{3}$ is $\omega=\left(1 / 2 \pi^{2}\right) \cdot$ $\sin ^{2} \theta_{1} \sin \theta_{2} d \theta_{1} d \theta_{2} d \theta_{3}$, where $0 \leqslant \theta_{1}, \theta_{2} \leqslant \pi, 0 \leqslant \theta_{3} \leqslant 2 \pi$.

If $g(q)=l q l^{-1}$ then $\operatorname{det}(1-t g)=(1-t)^{2}\left(1-2 t \cos 2 \theta_{1}+t^{2}\right)$. If $g(q)=-l q l^{-1}$ then $\operatorname{det}(1-t g)=(1+t)^{2}\left(1+2 t \cos 2 \theta_{1}+t^{2}\right)$. Therefore,

$$
\begin{aligned}
& \int_{A_{0}} F(\varphi(l, r)) d a_{0}=\int_{S^{3}} \frac{\left(1-t^{2}\right) \omega}{(1-t)^{2}\left(1-2 t \cos 2 \theta_{1}+t^{2}\right)}=\frac{1}{1-t}, \\
& \int_{A_{1}} F(\varphi(l, r)) d a_{1}=\int_{S^{3}} \frac{\left(1-t^{2}\right) \omega}{(1+t)^{2}\left(1+2 t \cos 2 \theta_{1}+t^{2}\right)}=\frac{1}{1+t} .
\end{aligned}
$$

Therefore, $F_{G}(t)=1 /\left(1-t^{2}\right)$.

5. Remarks about the Pólya function.

(1) $G$ acts on $S^{3}$ by $(g, x) \rightarrow g(x)$. We have:

Proposition. $F_{G}(t)=1$ if and only if $G$ acts transitively on $S^{3}$.

Proof. If $G$ acts transitively on $S^{3}$ and $f: S^{3} \rightarrow R$ is invariant under $G$ then $f$ is constant. Therefore

$$
\begin{aligned}
\operatorname{dim} H_{k}(G) & =0 \quad \text { if } k=0, \\
& =1 \quad \text { if } k \neq 0,
\end{aligned}
$$

and then $F_{G}(t)=1$.

Conversely, suppose that $F_{G}(t)=1$. Let $\bar{x}, \bar{y} \in S^{3}$ be arbitrary and $O_{\bar{x}}, O_{\bar{y}}$ the corresponding orbits. If $O_{\bar{x}} \cap O_{\bar{y}}=\varnothing$ then there exists a continuous function $f: S^{3} \rightarrow R$ such that $f\left(O_{\bar{x}}\right)=1$ and $f\left(O_{\bar{y}}\right)=0$. If $H(x)=\int_{G} f(g x) d g$ then $H$ is invariant under $G$ and $H(\bar{x})=1, H(\bar{y})=0$. Expand $H$ in spherical harmonics: $H=\Sigma_{k>0} f_{k}$. Then each $f_{k}$ is invariant under $G$. $F_{G}(t)=1$ implies that $f_{0}=$ constant, $f_{k}=0$ for $k>0$, and then $H$ is constant, a contradiction.

Remark. It is clear that $\left(S^{3} / C_{2} ; S^{3} / C_{2}\right)$ and $\left(S^{3} / C_{1} ; S^{3} / C_{1}\right)$ cannot act transitively on $S^{3}$. The other possible groups are of the form $G=\left(S^{3} / S^{3}\right.$; $M / M)$ and $O(4)$, and they indeed act transitively on $S^{3}$.

(2) A reflection $t: R^{n} \rightarrow R^{n}$ is called a simple reflection if $t$ leaves a hyperplane pointwise fixed; we have then $t^{2}=1$. A group $G$ of linear mappings of $R^{n}$ is a finite reflection group if it is finite and generated by simple reflections. Chevalley [4] proved that, for a finite reflection group $G, S(G)=$ $R\left[I_{1}, \cdots, I_{n}\right]$, where $S(G)$ denotes the algebra of polynomials invariant 
under $G$ and $I_{i}$ is a homogeneous invariant polynomial of degree $\left(m_{i}+1\right)$. It follows that the Pólya function is given by

$$
F_{G}(t)=\frac{1-t^{2}}{\left(1-t^{m_{1}+1}\right) \cdots\left(1-t^{m_{n}+1}\right)} \text {. }
$$

The groups $(O / T ; O / T)^{*},(O / V ; O / V)^{*},(I / I ; I / I)^{*},\left(I^{+} / C_{1} ; I / C_{1}\right)^{+*}$, $\left(T / C_{1} ; T / C_{1}\right)^{*},\left(O / C_{1} ; O / C_{1}\right)^{*},\left(I / C_{1} ; I / C_{1}\right)^{*}$ and $\left(C_{2 r} / C_{1} ; C_{2 r} / C_{1}\right)_{s=2 r-1 ; h=r}^{*}$ are finite reflection groups and the exponents $m_{i}$ are given in [5, p. 141].

About the pure subgroup of a finite reflection group we have the following result:

THEOREM. Let $G^{*}$ be a finite reflection group and $G=G^{*} \cap S O(4)$. Then $F_{G}(t)=\left(1+t^{d}\right) F_{G} *(t)$, with $d=\sum_{i=1}^{n} m_{i}$.

Proof. Let $S=R\left[x_{1}, \cdots, x_{n}\right], S(G)$ be the algebra of polynomials invariant under $G, S\left(G^{*}\right)=R\left[I_{1}, \cdots, I_{n}\right]$, with degree of $I_{1}$ equal to $\left(m_{i}+1\right)$. Let $\bar{S}, \overline{S(G)}, \overline{S\left(G^{*}\right)}$, denote the corresponding quotient fields. Then $S$ is integral over $S\left(G^{*}\right), \overline{S\left(G^{*}\right)}$ is integrally closed, $\overline{S(G)}=\bar{S}(G)$; Then $\bar{S} \supset \overline{S(G)}$ is a Galois extension. We have $\bar{S}(G)=\bar{S}\left(G^{*}\right)[J]$ where $J \in S(G)-S\left(G^{*}\right), J^{2} \in S\left(G^{*}\right)$ and we can take $J^{2}$ square free and homogeneous.

Suppose $(P+Q J) / R \in S(G)$, where $P, Q, R \in S\left(G^{*}\right)$. Then the norm and the trace of this element belong to $S\left(G^{*}\right)$, and we conclude that $R$ divides $P$ and $R$ divides $Q$. Therefore $S(G)=S\left(G^{*}\right) \oplus S\left(G^{*}\right) J$. If the degree of $J$ is $d$ then we get the result. We have $d=\Sigma m_{i}$ because $F_{G}(1 / t)=(-1)^{n} t^{n} F_{G}(t)$.

(3) In the table of Pólya functions that follows we use the following notations and results:

(a) $S(m, \alpha)=\sum_{k=0}^{m-1} \frac{1-t^{2}}{m\left[1-2 t \cos (2 k \pi / m-\alpha)+t^{2}\right]\left[1-2 t \cos (2 k \pi / m+\alpha)+t^{2}\right]}$,

(b) $\quad T(m, \beta)=\sum_{k=0}^{m-1} \frac{1-t^{2}}{m\left[1+2 t \cos (2 k \pi / m+\beta)+t^{2}\right]}$.

By the same technique used in the proof of the lemma on p. 8 we can prove that

$$
\begin{aligned}
S(m, \alpha) & =\frac{2 t^{m}\left(1-t^{2}\right) \cos \alpha \sin m \alpha+\sin \alpha\left(1+t^{2}\right)\left(1-t^{2 m}\right)}{\sin \alpha\left(1-2 t^{2} \cos 2 \alpha+t^{4}\right)\left(1-2 t^{m} \cos m \alpha+t^{2 m}\right)} \\
T(m, \beta) & =\frac{1-t^{2 m}}{1-2 t^{m} \cos m \beta+t^{2 m}} \quad \text { if } m \text { is even, } \\
& =\frac{1-t^{2 m}}{1+2 t^{m} \cos m \beta+t^{2 m}} \quad \text { if } m \text { is odd }
\end{aligned}
$$


(c) $A_{s}(2 m, 2 n, r)=\sum_{k=0}^{2 m-1} \sum_{n=0}^{2 n-1} \sum_{j=0}^{r-1} \frac{1-t^{2}}{4 m n r\left(1-2 t \cos \alpha+t^{2}\right)\left(1-2 t \cos \beta+t^{2}\right)}$, where

$$
\alpha=\frac{(n-m s) j+r(n k-m h)}{m n r} \pi, \quad \beta=\frac{(n+m s) j+r(n k+m h)}{m n r} \pi .
$$

$$
B(2 m, 2 n)=A_{s}(2 m, 2 n, 1)
$$

(d)

$$
=\sum_{k=0}^{2 m-1} \sum_{n=0}^{2 n-1} \frac{1-t^{2}}{4 m n\left(1-2 t \cos \alpha+t^{2}\right)\left(1-2 t \cos \beta+t^{2}\right)}
$$

with $\alpha=(n k-m h) \pi / m n, \beta=(n k+m h) \pi / m n$. In particular, we have

$$
B(2 m, 2 m)=\left(1+6 t^{2 m}+t^{4 m}\right) /\left(1-t^{2}\right)\left(1-t^{2 m}\right)^{2} .
$$

$C(2 m, 2 n)=2 A_{1}(2 m, 2 n, 2)-B(2 m, 2 n)$

(e) $=\sum_{k=1}^{2 m-1} \sum_{n=0}^{2 n-1} \frac{1-t^{2}}{4 m n\left[1-2 t \cos \left(\frac{2 k+1}{2 m} \pi-\frac{2 h+1}{2 n} \pi\right)+t^{2}\right]\left[1-2 t \cos \left(\frac{2 k+1}{2 m} \pi+\frac{2 h+1}{2 m} \pi\right)+t^{2}\right]}$.

In particular, $C(2 m, 2 m)=1 /\left(1-t^{2}\right)$.

$$
D(n, 2 r, s, h, k)
$$

(f) $=\sum_{\alpha=0}^{n-1} \sum_{\beta=0}^{n-1} \sum_{j=0}^{2 r-1}\left(2 n^{2} r\left[1+2 t \cos \frac{2 r(\alpha+\beta)+1 / 2(h-k)+(1+s) j}{n r} \pi+t^{2}\right]\right)^{-1}$.

(g) $E_{s}(m, n, 2 r)=\sum_{k=0}^{m-1} \sum_{n=0}^{n-1} \sum_{j=0}^{2 r-1} \frac{1-t^{2}}{2 m n r\left(1-2 t \cos \alpha+t^{2}\right)\left(1-2 t \cos \beta+t^{2}\right)}$,

where

$$
\alpha=\frac{(n-m s) j+2 r(k n-m h)}{m n r} \pi, \quad \beta=\frac{(n+m s) j+2 r(k n+m h)}{m n r} \pi .
$$

$$
H(n, 2 r, s, h, k)
$$

(h) $=\sum_{\alpha=0}^{n-1} \sum_{\beta=0}^{n-1} \sum_{j=0}^{2 r-1}\left(2 n^{2} r\left[1-2 t \cos \frac{(1-s) j+2 r(\alpha-\beta)-1 / 2(h+k)}{n r} \pi+t^{2}\right]\right)^{-1}$. 
(4) If $F_{G}(t)=\Sigma_{k>0} a_{k} t^{k}$ is such that $a_{k}=0$ for $k$ odd, then it follows from the results of [8] that $G$ cannot be the group of symmetries of a hypersurface of constant width. An example is $(I / I ; I / I)$ in contrast with the icosahedral group in $\mathbf{R}^{\mathbf{3}}$.

6. Polya functions of the closed subgroups of $O(4)$.

Group

Pólya function

(1) $\quad\left(S^{3} / S^{3} ; S^{3} / S^{3}\right)=S O(4)$

(2) $\left(S^{3} / C_{2} ; S^{3} / C_{2}\right)$

1

(3) $\quad\left(S^{3} / C_{1} ; S^{3} / C_{1}\right)$

$\frac{1}{1-t^{2}}$

$\frac{1}{1-t}$

(4) $\left(S^{3} / S^{3} ; H_{2} / H_{2}\right)$

1

(5) $\left(S^{3} / S^{3} ; H_{1} / H_{1}\right)$

1

(6) $\left(S^{3} / S^{3} ; I / I\right)$

1

(7) $\left(S^{3} / S^{3} ; O / O\right)$

1

(8) $\left(S^{3} / S^{3} ; T / T\right)$

1

(9) $\left(S^{3} / S^{3} ; D_{n} / D_{n}\right)$

1

(10) $\left(S^{3} / S^{3} ; C_{2 n} / C_{2 n}\right)$

1

(11) $\left(\mathrm{H}_{2} / \mathrm{H}_{2} ; \mathrm{H}_{2} / \mathrm{H}_{2}\right)$

$\frac{1}{1-t^{4}}$

(12) $\left(H_{2} / H_{1} ; H_{2} / H_{1}\right)$

$\frac{1}{1-t^{2}}$

(13) $\left(H_{2} / H_{2} ; H_{1} / H_{1}\right)$

$\frac{1}{1-t^{4}}$

(14) $\left(H_{2} / H_{1} ; O / T\right)$

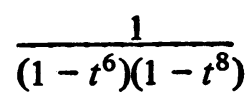

(15) $\left(H_{2} / H_{1} ; D_{n} / C_{2 n}\right)$

$$
\frac{1}{\left(1-t^{2}\right)\left(1-t^{2 n}\right)}
$$

(16) $\left(H_{2} / H_{1} ; D_{2 n} / D_{n}\right)$

$$
\begin{cases}\frac{1}{\left(1-t^{4}\right)\left(1-t^{2 n}\right)} & \text { if } n \text { is odd } \\ \frac{1+t^{2 n+2}}{\left(1-t^{4}\right)\left(1-t^{4 n}\right)} & \text { if } n \text { is even }\end{cases}
$$


Group

(17)

$$
\left(H_{2} / H_{1} ; C_{2 n} / C_{n}\right)
$$

$\left(\mathrm{H}_{2} / \mathrm{H}_{2} ; I / I\right)$

(18)

(19)

(20)

(21)

$$
\left(H_{2} / H_{2} ; D_{n} / D_{n}\right)
$$

$\left(\mathrm{H}_{2} / \mathrm{H}_{2} ; \mathrm{O} / \mathrm{O}\right)$,

$\left(H_{2} / H_{2} ; T / T\right)$,

Polya function

$$
\begin{cases}\frac{1+2 t^{n+2}+t^{2 n}}{\left(1-t^{4}\right)\left(1-t^{2 n}\right)} & \text { if } n \equiv 0(\bmod 4) \\ \frac{1+2 t^{2 n+2}+t^{4 n}}{\left(1-t^{4}\right)\left(1-t^{4 n}\right)} & \text { if } n \equiv 1 \text { or } 3(\bmod 4) \\ \frac{1+t^{n}}{\left(1-t^{2}\right)\left(1-t^{n}\right)} & \text { if } n \equiv 2(\bmod 4)\end{cases}
$$

$$
\frac{1}{\left(1-t^{12}\right)\left(1-t^{20}\right)}
$$

$$
\frac{1}{\left(1-t^{8}\right)\left(1-t^{12}\right)}
$$

$$
\frac{1+t^{12}}{\left(1-t^{8}\right)\left(1-t^{12}\right)}
$$

$$
\begin{cases}\frac{1+t^{2 n+2}}{\left(1-t^{4}\right)\left(1-t^{4 n}\right)} & \text { if } n \text { is odd } \\ \frac{1}{\left(1-t^{4}\right)\left(1-t^{2 n}\right)} & \text { if } n \text { is even }\end{cases}
$$

$$
\frac{1+t^{2 n}}{\left(1-t^{4}\right)\left(1-t^{2 n}\right)}
$$

$$
\frac{1}{\left(1-t^{2}\right)\left(1-t^{n}\right)}
$$

$$
\frac{1}{1-t^{2}}
$$

$$
\frac{1+t^{n}}{\left(1-t^{2}\right)\left(1-t^{n}\right)}
$$

$\frac{1-2 t^{4}-t^{6}+t^{8}+t^{10}+t^{12}+t^{14}-t^{16}-2 t^{18}+t^{22}}{\left(1-t^{4}\right)^{2}\left(1-t^{6}\right)\left(1-t^{10}\right)}$

$$
\frac{1+t^{18}}{\left(1-t^{8}\right)\left(1-t^{12}\right)}
$$

$$
\frac{1+t^{12}}{\left(1-t^{6}\right)\left(1-t^{8}\right)}
$$

$$
\frac{1+t^{2 n+2}}{\left(1-t^{4}\right)\left(1-t^{2 n}\right)}
$$


Group

(30) $\quad\left(H_{1} / H_{1} ; C_{2 n} / C_{2 n}\right)$

(31)

$$
\left\{\begin{array}{l}
\left(C_{2 m} / C_{2 m} ; D_{n} / D_{n}\right) \\
\left(C_{2 m} / C_{2 m} ; D_{m} / D_{m}\right)
\end{array}\right.
$$

$$
\left(C_{2 m r} / C_{2 m} ; C_{2 n r} / C_{2 n}\right)_{s}
$$

$\left(C_{2 m} / C_{2 m} ; C_{2 m} / C_{2 m}\right)_{s}$

$$
\left\{\begin{array}{l}
\left(C_{4 m} / C_{2 m} ; D_{n} / C_{2 n}\right) \\
\left(C_{4 m} / C_{2 m} ; D_{m} / C_{2 m}\right)
\end{array}\right.
$$

$$
\left(C_{2 m} / C_{2 m} ; I / I\right)
$$

$$
\left(C_{4 m} / C_{2 m} ; D_{2 n} / D_{n}\right)
$$

$$
\left(C_{4 m} / C_{2 m} ; D_{2 m} / D_{m}\right)
$$$$
\left(C_{2 m} / C_{2 m} ; T / T\right)
$$$$
\left(C_{6 m} / C_{2 m} ; T / V\right)
$$

$$
\left(C_{2 m} / C_{2 m} ; 0 / 0\right)
$$$$
\left(C_{4 m} / C_{2 m} ; O / T\right)
$$

Pólya function

$$
\begin{aligned}
& \frac{1+t^{2 n}}{\left(1-t^{2}\right)\left(1-t^{2 n}\right)} \\
& A_{s}(2 m, 2 n, r)
\end{aligned}
$$

$$
\frac{1+6 t^{2 m}+t^{4 m}}{\left(1-t^{2}\right)\left(1-t^{2 m}\right)^{2}}
$$

$1 / 2 B(2 m, 2 n)+1 / 2 S(2 m, \pi / 2)$

$\left\{\begin{array}{l}\frac{1+3 t^{2 m}+3 t^{2 m+2}+t^{4 m+2}}{\left(1-t^{4}\right)\left(1-t^{2 m}\right)^{2}} \text { if } m \text { is even } \\ \frac{1+2 t^{2 m}+5 t^{2 m+2}+5 t^{4 m}+2 t^{4 m+2}+t^{6 m+2}}{\left(1-t^{4}\right)\left(1-t^{2 m}\right)\left(1-t^{4 m}\right)} \\ \text { if } m \text { is odd }\end{array}\right.$

$1 / 2 B(2 m, 2 n)+S(4 m, \pi / 2)-1 / 2 S(2 m, \pi / 2)$

$\left\{\begin{array}{c}\frac{1+2 t^{2 m}+5 t^{2 m+2}+5 t^{4 m}+2 t^{4 m+2}+t^{6 m+2}}{\left(1-t^{4}\right)\left(1-t^{2 m}\right)\left(1-t^{4 m}\right)} \\ \text { if } m \text { is even } \\ \frac{1+4 t^{2 m}+3 t^{2 m+2}+3 t^{4 m}+4 t^{4 m+2}+t^{6 m+2}}{\left(1-t^{4}\right)\left(1-t^{2 m}\right)\left(1-t^{4 m}\right)} \\ \text { if } m \text { is odd }\end{array}\right.$

$1 / 4 B(2 m, 2 n)+1 / 4 C(2 m, 2 n)+1 / 2 S(4 m, \pi / 2)$

$\frac{1+t^{2 m}+2 t^{2 m+2}+2 t^{4 m}+t^{4 m+2}+t^{6 m+2}}{\left(1-t^{4}\right)\left(1-t^{2 m}\right)\left(1-t^{4 m}\right)}$

$\frac{1}{12} S(2 m, 0)+\frac{2}{3} S\left(2 m, \frac{\pi}{3}\right)+\frac{1}{4} S\left(2 m, \frac{\pi}{2}\right)$

$\frac{1}{12} S(2 m, 0)+\frac{1}{4} S\left(2 m, \frac{\pi}{2}\right)+S\left(6 m, \frac{\pi}{3}\right)-\frac{1}{3} S\left(2 m, \frac{\pi}{3}\right)$

$\frac{3}{8} s\left(2 m, \frac{\pi}{2}\right)+\frac{1}{4} s\left(2 m, \frac{\pi}{4}\right)+\frac{1}{24} s(2 m, 0)+\frac{1}{3} s\left(2 m, \frac{\pi}{3}\right)$

$\frac{1}{2} S\left(4 m, \frac{\pi}{2}\right)-\frac{1}{8} S\left(2 m, \frac{\pi}{2}\right)+\frac{1}{2} S\left(4 m, \frac{\pi}{4}\right)$

$$
-\frac{1}{4} S\left(2 m, \frac{\pi}{4}\right)+\frac{1}{24} S(2 m, 0)+\frac{1}{3} S\left(2 m, \frac{\pi}{3}\right)
$$

$$
\frac{1}{60} S(2 m, 0)+\frac{1}{10} S\left(2 m, \frac{2 \pi}{5}\right)+\frac{1}{10} S\left(2 m, \frac{3 \pi}{5}\right)
$$

$$
\begin{aligned}
& +\frac{1}{3} S\left(2 m, \frac{\pi}{3}\right)+\frac{1}{10} S\left(2 m, \frac{\pi}{5}\right) \\
& +\frac{1}{10} S\left(2 m, \frac{4 \pi}{5}\right)+\frac{1}{4} S\left(2 m, \frac{\pi}{2}\right)
\end{aligned}
$$


SYMMETRIES OF SPHERICAL HARMONICS

173

Group

$$
\left(D_{m} / D_{m} ; D_{n} / D_{n}\right)
$$

$$
\left(D_{m} / D_{m} ; D_{m} / D_{m}\right)
$$

$$
\left(D_{m r} / C_{2 m} ; D_{n r} / C_{2 n}\right)_{s}
$$

$$
\begin{aligned}
& \left(D_{m} / C_{2 m} ; D_{m} / C_{2 m}\right)_{s} \\
& \left(D_{2 m} / D_{m} ; D_{2 n} / D_{n}\right)
\end{aligned}
$$

(44) $\quad\left(D_{m} / D_{m}, T / T\right)$

(45) $\quad\left(D_{m} / D_{m} ; O / O\right)$

(46) $\quad\left(D_{m} / C_{2 m} ; O / T\right)$

(47)

$$
\left(D_{2 m} / D_{m} ; O / T\right)
$$

Pólya function

$$
\frac{1}{4} B(2 m, 2 n)+\frac{1}{4} S\left(2 m, \frac{\pi}{2}\right)+\frac{1}{4} S\left(2 n, \frac{\pi}{2}\right)+\frac{1}{4} \frac{1}{1-t^{2}}
$$$$
\left\{\begin{array}{l}
\frac{1+t^{2 m}+t^{2 m+2}+t^{4 m+2}}{\left(1-t^{4}\right)\left(1-t^{2 m}\right)^{2}} \text { if } m \text { is even } \\
\frac{1+3 t^{2 m+2}+3 t^{4 m}+t^{6 m+2}}{\left(1-t^{4}\right)\left(1-t^{2 m}\right)\left(1-t^{4 m}\right)} \text { if } m \text { is odd }
\end{array}\right.
$$$$
\frac{1}{2} A_{s}(2 m, 2 n, r)+\frac{1}{2} \frac{1}{1-t^{2}}
$$

$$
\begin{aligned}
& \frac{\left(1+t^{2 m}\right)^{2}}{\left(1-t^{2}\right)\left(1-t^{2 m}\right)^{2}} \\
& \frac{1}{8} B(2 m, 2 n)+\frac{1}{8} C(2 m, 2 n)+\frac{1}{4} S\left(4 m, \frac{\pi}{2}\right) \\
& \quad+\frac{1}{4} S\left(4 n, \frac{\pi}{2}\right)+\frac{1}{4} \frac{1}{1-t^{2}}
\end{aligned}
$$

$$
\frac{\left(1+t^{2 m+2}\right)\left(1+t^{4 m}\right)}{\left(1-t^{4}\right)\left(1-t^{2 m}\right)\left(1-t^{4 m}\right)}
$$$$
\frac{1}{4} B(2 m, 2 n)+\frac{1}{4} S\left(2 n, \frac{\pi}{2}\right)+\frac{1}{2} S\left(4 m, \frac{\pi}{2}\right)
$$$$
-\frac{1}{4} S\left(2 m, \frac{\pi}{2}\right)+\frac{1}{4} \frac{1}{1-t^{2}}
$$$$
\frac{1+t^{2 m}+2 t^{2 m+2}+2 t^{4 m}+t^{4 m+2}+t^{6 m+2}}{\left(1-t^{4}\right)\left(1-t^{2 m}\right)\left(1-t^{4 m}\right)}
$$

$$
\begin{gathered}
\frac{1}{24} S(2 m, 0)+\frac{1}{3} S\left(2 m, \frac{\pi}{3}\right)+\frac{1}{8} S\left(2 m, \frac{\pi}{2}\right) \\
+\frac{1}{2} \frac{1+t^{12}}{\left(1+t^{6}\right)\left(1-t^{8}\right)}
\end{gathered}
$$

$$
\begin{aligned}
& \frac{1}{48} S(2 m, 0)+\frac{1}{6} S\left(2 m, \frac{\pi}{3}\right)+\frac{3}{16} S\left(2 m, \frac{\pi}{2}\right) \\
& \quad+\frac{1}{8} S\left(2 m, \frac{\pi}{4}\right)+\frac{1}{2}\left(\frac{1-t^{18}}{\left(1-t^{8}\right)\left(1-t^{12}\right)}\right) \\
& \frac{1}{24} S(2 m, 0)+\frac{1}{3}\left(2 m, \frac{\pi}{3}\right)+\frac{1}{8} S\left(2 m, \frac{\pi}{2}\right) \\
& \quad+\frac{1}{2} \frac{1+t^{6}}{1-t^{8}}
\end{aligned}
$$

$$
\begin{aligned}
\frac{1}{48} & S(2 m, 0)+\frac{1}{4} S\left(4 m, \frac{\pi}{2}\right)-\frac{1}{16} S\left(2 m, \frac{\pi}{2}\right) \\
& +\frac{1}{6} S\left(2 m, \frac{\pi}{3}\right)+\frac{1}{4} S\left(4 m, \frac{\pi}{4}\right)-\frac{1}{8} s\left(2 m, \frac{\pi}{4}\right) \\
& +\frac{1}{2} \frac{1-t^{18}}{\left(1-t^{8}\right)\left(1-t^{12}\right)}
\end{aligned}
$$


Group

(48)

(49)

(50)

(54)

(60)

$\left(D_{m} / D_{m} ; I / I\right)$

$\left(D_{3 m} / C_{2 m} ; O / V\right)$

$(T / T ; T / T)$

$\left(T / C_{2} ; T / C_{2}\right)$

$(T / V ; T / V)$

$(T / T ; O / O)$

$(T / T ; I / I)$

$(0 / 0 ; 0 / 0)$

$\left(O / C_{2} ; O / C_{2}\right)$

$(O / V ; O / V)$

$(O / T ; O / T)$

$(O / O ; I / I)$

$(I / I ; I / I)$

(61)
Pólya function

$$
\begin{aligned}
\frac{1}{24} & S(2 m, 0)+\frac{1}{8} S\left(2 m, \frac{\pi}{2}\right)+\frac{1}{2} S\left(6 m, \frac{\pi}{3}\right) \\
& -\frac{1}{6} S\left(2 m, \frac{\pi}{3}\right)+\frac{1}{2} \frac{1+t^{6}}{1-t^{8}} \\
\frac{1}{120} & S(2 m, 0)+\frac{1}{8} S\left(2 m, \frac{\pi}{2}\right)+\frac{1}{6} s\left(2 m, \frac{\pi}{3}\right) \\
& +\frac{1}{20} S\left(2 m, \frac{\pi}{5}\right)+\frac{1}{20} S\left(2 m, \frac{2 \pi}{5}\right) \\
& +\frac{1}{20} S\left(2 m, \frac{3 \pi}{5}\right)+\frac{1}{20} S\left(2 m, \frac{4 \pi}{5}\right) \\
& +\frac{1}{2} \frac{1-t^{30}}{\left(1-t^{12}\right)\left(1-t^{20}\right)}
\end{aligned}
$$$$
\frac{\left(1-t^{4}+t^{8}\right)\left(1+t^{12}\right)}{\left(1-t^{4}\right)\left(1-t^{6}\right)\left(1-t^{12}\right)}
$$$$
\frac{\left(1+t^{4}\right)\left(1-t^{2}+t^{4}\right)}{\left(1-t^{2}\right)^{2}\left(1-t^{6}\right)}
$$$$
\frac{1+t^{12}}{\left(1-t^{4}\right)^{2}\left(1-t^{6}\right)}
$$

$\frac{1-t^{6}-t^{8}+2 t^{12}+t^{14}-2 t^{20}+2 t^{24}-t^{30}-2 t^{32}+t^{36}+t^{38}-t^{44}}{\left(1-t^{6}\right)\left(1-t^{8}\right)^{2}\left(1-t^{24}\right)}$

$\frac{1+t^{12}+t^{20}+t^{24}+2 t^{30}+t^{36}+t^{40}+t^{48}+t^{60}}{\left(1-t^{12}\right)\left(1-t^{20}\right)\left(1-t^{30}\right)}$

$\frac{1-t^{6}+t^{12}+t^{24}-t^{30}+t^{36}}{\left(1-t^{6}\right)\left(1-t^{8}\right)\left(1-t^{24}\right)}$

$\frac{1+t^{10}}{\left(1-t^{2}\right)\left(1-t^{4}\right)\left(1-t^{6}\right)}$

$$
\frac{1+t^{16}}{\left(1-t^{4}\right)\left(1-t^{6}\right)\left(1-t^{8}\right)}
$$

$\frac{1+t^{24}}{\left(1-t^{6}\right)\left(1-t^{8}\right)\left(1-t^{12}\right)}$ 
Group

Pólya function

(62) $\left(I^{+} / C_{2} ; I / C_{2}\right)^{+}$

$\frac{\left(1+t^{8}\right)\left(1+t^{10}\right)}{\left(1-t^{4}\right)\left(1-t^{6}\right)\left(1-t^{10}\right)}$

$$
\left(C_{2 m r} / C_{m} ; C_{2 n r} / C_{n}\right)_{s} \quad E_{s}(m, n, 2 r)
$$

(63)

$$
m \equiv n \equiv 1(\bmod 2)
$$

$\left(C_{2 r} / C_{1} ; C_{2 r} / C_{1}\right)_{s=2 r-1} \quad \frac{1+t^{r}}{(1-t)^{2}\left(1-t^{r}\right)}$

$\left(D_{m r} / C_{m} ; D_{n r} / C_{n}\right)_{s}$

$\frac{1}{2} E_{s}(m, n, 2 r)+\frac{1}{2} \frac{1}{1-t^{2}}$

(64)

$$
\left(D_{r} / C_{1} ; D_{r} / C_{1}\right)_{s=2 r-1} \quad \frac{1+t^{r+1}}{(1-t)\left(1-t^{2}\right)\left(1-t^{r}\right)}
$$

(65) $\quad\left(T / C_{1} ; T / C_{1}\right)$

$$
\frac{1+t^{6}}{(1-t)\left(1-t^{3}\right)\left(1-t^{4}\right)}
$$

(66) $\left(O / C_{1} ; O / C_{1}\right)_{1}$

$$
\frac{1+t^{9}}{(1-t)\left(1-t^{4}\right)\left(1-t^{6}\right)}
$$

(67) $\quad\left(O / C_{1} ; O / C_{1}\right)_{2}$

$$
\frac{1+t^{7}}{\left(1-t^{2}\right)\left(1-t^{3}\right)\left(1-t^{4}\right)}
$$

(68) $\quad\left(I / C_{1}: I / C_{1}\right)$

$$
\frac{1+t^{15}}{(1-t)\left(1-t^{6}\right)\left(1-t^{10}\right)}
$$

(69) $\left(I^{+} / C_{1} ; I / C_{1}\right)^{+}$

$$
\frac{1+t^{10}}{\left(1-t^{3}\right)\left(1-t^{4}\right)\left(1-t^{5}\right)}
$$

(70) $\quad\left(S^{3} / S^{3} ; S^{3} / S^{3}\right)^{*}=O(4)$

(71) $\left(S^{3} / C_{2} ; S^{3} / C_{2}\right)^{*}$

$$
\frac{1}{1-t^{2}}
$$

(72) $\quad\left(S^{3} / C_{1} ; S^{3} / C_{1}\right)_{1}^{*}$

$\frac{1}{1-t}$

(73) $\quad\left(S^{3} / C_{1} ; S^{3} / C_{1}\right)_{2}^{*}$

$\frac{1}{1-t^{2}}$

(74) $\left(\mathrm{H}_{2} / \mathrm{H}_{2} ; \mathrm{H}_{2} / \mathrm{H}_{2}\right)^{*}$

$\frac{1}{1-t^{4}}$

(75) $\quad\left(H_{2} / H_{1} ; H_{2} / H_{1}\right)_{1}^{*}$

$\frac{1}{1-t^{2}}$

(76) $\quad\left(H_{2} / H_{1} ; H_{2} / H_{1}\right)_{2}^{*}$ 
(77) $\quad\left(H_{2} / C_{n} ; H_{2} / C_{n}\right)_{1}^{*}$

$$
\frac{1}{\left(1-t^{2}\right)\left(1-t^{2 n}\right)}
$$

(78) $\quad\left(H_{2} / C_{n} ; H_{2} / C_{n}\right)_{2}^{*}$

$$
\frac{1}{\left(1-t^{2}\right)\left(1-t^{n}\right)}
$$

(79) $\left(H_{1} / H_{1} ; H_{1} / H_{1}\right)^{*}$

$$
\frac{1}{1-t^{2}}
$$

(80) $\quad\left(H_{1} / C_{n} ; H_{1} / C_{n}\right)_{1}^{*}$

$$
\frac{1}{\left(1-t^{2}\right)\left(1-t^{n}\right)}
$$

(81) $\quad\left(H_{1} / C_{n} ; H_{1} / C_{n}\right)_{2}^{*}$

$$
\frac{1+t^{2 n}}{\left(1-t^{2}\right)\left(1-t^{2 n}\right)}
$$

$$
\left(C_{2 n r} / C_{n} ; C_{2 n r} / C_{n}\right)_{s, h}^{*} \quad 1 / 2 E_{s}(n, n, 2 r)+1 / 2 D(n, 2 r, s, 2 h, 0)
$$

$$
\left(C_{2 r} / C_{1} ; C_{2 r} / C_{1}\right)_{s=2 r-1 ; h=r} \frac{1}{\left(1-t^{2}\right)\left(1-t^{r}\right)}
$$

(84) $\quad\left(D_{n} / D_{n} ; D_{n} / D_{n}\right)^{*}$

$$
\begin{cases}\frac{1+t^{2 n+2}}{\left(1-t^{4}\right)\left(1-t^{2 n}\right)^{2}} & \text { if } n \text { is even } \\ \frac{1+2 t^{2 n+2}+t^{4 n}}{\left(1-t^{4}\right)\left(1-t^{2 n}\right)\left(1-t^{4 n}\right)} & \text { if } n \text { is odd }\end{cases}
$$

$$
\begin{aligned}
& \left(D_{n r} / C_{n} ; D_{n r} / C_{n}\right)_{s, h, k}^{*} \\
& \frac{1}{2} D(n, r, s, h, k)+\frac{1}{4} E_{s}(n, n, 2 r) \\
& +\frac{1}{4} \frac{1}{1-t^{2}}+\frac{1}{4} H(n, 2 r, s, h, k)
\end{aligned}
$$

$$
\left(D_{r} / C_{1} ; D_{r} / C_{1}\right)_{s=1 ; n=k=r}^{*} \begin{cases}\frac{1+t^{r+1}}{\left(1-t^{2}\right)^{2}\left(1-t^{7}\right)} & \text { if } r \text { is even } \\ \frac{1+t^{r+1}+t^{r+2}+t^{2 r+1}}{\left(1-t^{2}\right)^{2}\left(1-t^{2 r}\right)} & \text { if } r \text { is odd }\end{cases}
$$

$$
\left(D_{n r} / C_{n} ; D_{n r} / C_{n}\right)_{s, h, k,-}^{*} \quad \frac{1}{4} E_{s}(n, n, 2 r)+\frac{3-t^{2}}{4\left(1-t^{4}\right)}
$$

$$
\begin{array}{ll}
\left(D_{2 n} / D_{n} ; D_{2 n} / D_{n}\right)^{*} & \frac{1+t^{2 n+2}}{\left(1-t^{4}\right)\left(1-t^{2 n}\right)\left(1-t^{4 n}\right)} \\
\left(D_{2 n} / D_{n} ; D_{2 n} / D_{n}\right)_{-}^{*} & \frac{1-t^{2 n}+t^{4 n}+t^{4 n+2}}{\left(1-t^{4}\right)\left(1-t^{2 n}\right)\left(1-t^{4 n}\right)}
\end{array}
$$


Group

$\begin{array}{ll}\text { (89) } & \left(T / C_{2} ; T / C_{2}\right)_{c}^{*} \\ \text { (90) } & \left(T / C_{2} ; T / C_{2}\right)^{*}\end{array}$

(91) $\quad(T / V ; T / V)^{*}$

(92) $\quad(T / V ; T / V)^{*}$

(93) $\quad(T / T ; T / T)^{*}$

(94) $\left(O / C_{2} ; O / C_{2}\right)^{*}$

(95) $\quad(O / T ; O / T)^{*}$

(96) $\quad(O / T ; O / T)^{*}$

(97) $(O / V ; O / V)^{*}$

(98) $\quad(0 / 0 ; 0 / 0)^{*}$

(99) $\left(I / C_{2} ; I / C_{2}\right)^{*}$

(100) $\quad(I / I ; I / I)^{*}$

(101) $\left(I^{+} / C_{2} ; I / C_{2}\right)^{+*}$

(102) $\quad\left(T / C_{1} ; T / C_{1}\right)_{c}^{*}$

(103) $\quad\left(T / C_{1} ; T / C_{1}\right)_{c-}^{*}$

(104) $\left(T / C_{1} ; T / C_{1}\right)^{*}$
Pólya function

$$
\begin{aligned}
& \frac{1+t^{6}}{\left(1-t^{2}\right)\left(1-t^{4}\right)\left(1-t^{6}\right)} \\
& \frac{1+t^{4}}{\left(1-t^{2}\right)\left(1-t^{4}\right)\left(1-t^{6}\right)}
\end{aligned}
$$$$
\frac{1+t^{12}}{\left(1-t^{4}\right)\left(1-t^{6}\right)\left(1-t^{8}\right)}
$$$$
\frac{1+t^{4}}{\left(1-t^{4}\right)\left(1-t^{6}\right)\left(1-t^{8}\right)}
$$

$$
\frac{1+t^{12}}{\left(1-t^{6}\right)\left(1-t^{8}\right)\left(1-t^{12}\right)}
$$

$$
\frac{1}{\left(1-t^{2}\right)\left(1-t^{4}\right)\left(1-t^{6}\right)}
$$

$\frac{1}{\left(1-t^{6}\right)\left(1-t^{8}\right)\left(1-t^{12}\right)}$

$$
\frac{1-t^{6}+t^{18}}{\left(1-t^{6}\right)\left(1-t^{8}\right)\left(1-t^{12}\right)}
$$

$\frac{1}{\left(1-t^{4}\right)\left(1-t^{6}\right)\left(1-t^{8}\right)}$

$\frac{1+t^{18}}{\left(1-t^{8}\right)\left(1-t^{12}\right)\left(1-t^{24}\right)}$

$$
\frac{1}{\left(1-t^{2}\right)\left(1-t^{6}\right)\left(1-t^{10}\right)}
$$

$\frac{1}{\left(1-t^{12}\right)\left(1-t^{20}\right)\left(1-t^{30}\right)}$

$\frac{1+t^{8}}{\left(1-t^{4}\right)\left(1-t^{6}\right)\left(1-t^{10}\right)}$

$\frac{1+t^{6}}{(1-t)\left(1-t^{4}\right)\left(1-t^{6}\right)}$

$\frac{1+t^{6}}{\left(1-t^{2}\right)\left(1-t^{4}\right)\left(1-t^{3}\right)}$

$\frac{1}{(1-t)\left(1-t^{3}\right)\left(1-t^{4}\right)}$ 


\section{Group}

(105) $\left(T / C_{1} ; T / C_{1}\right)^{*}$

(106) $\left(O / C_{1} ; O / C_{1}\right)^{*}$

(107) $\left(O / C_{1} ; O / C_{1}\right)_{-}^{*}$

(108) $\left(I / C_{1} ; I / C_{1}\right)^{*}$

(109) $\left(I / C_{1} ; I / C_{1}\right)^{*}$

(110)- $\left(I^{+} / C_{1} ; I / C_{1}\right)^{+*}$

(111) $\left(I^{+} / C_{1} ; I / C_{1}\right)_{-}^{+*}$
Pólya function

$\frac{1-t^{3}+t^{4}+t^{6}}{\left(1-t^{2}\right)\left(1-t^{3}\right)\left(1-t^{4}\right)}$

$\frac{1}{(1-t)\left(1-t^{4}\right)\left(1-t^{6}\right)}$

$\frac{1+t^{9}}{\left(1-t^{2}\right)\left(1-t^{4}\right)\left(1-t^{6}\right)}$

$\frac{1}{(1-t)\left(1-t^{6}\right)\left(1-t^{10}\right)}$

$\frac{1+t^{15}}{\left(1-t^{2}\right)\left(1-t^{6}\right)\left(1-t^{10}\right)}$

$\frac{1}{\left(1-t^{3}\right)\left(1-t^{4}\right)\left(1-t^{5}\right)}$

$\frac{1-t^{5}+t^{8}+t^{10}}{\left(1-t^{4}\right)\left(1-t^{5}\right)\left(1-t^{6}\right)}$

\section{REFERENCES}

1. J. F. Adams, Lectures on Lie groups, Benjamin, New York, 1969. MR 40 \#5780.

2. N. Bourbaki, Éléments de mathématique, Fasc. XXXIV. Groupes et algèbres de Lie. Chaps. IV, V, VI, Actualités Sci. Indust., no. 1337, Hermann, Paris, 1968 . MR 39 \#1590.

3. - Éléments de mathématique. Fasc. XXX. Algebre commutative. Chaps. 5, 6, Actualités Sci. Indust., no. 1308, Hermann, Paris, 1964. MR 33 \#2660.

4. C. Chevalley, Invariants of finite groups generated by reflections, Amer. J. Math. 77 (1955), 778-782. MR 17, 345, 1436.

5. H. S. M. Coxeter and W. O. Moser, Generators and relations for discrete groups, 2nd ed., Ergebnisse der Mathematik und ihrer Grenzgebiete, N. F., Band 14, Springer-Verlag, Berlin and New York, 1965. MR 30 \#4818.

6. J. Dieudonné, Éléments d'analyse. Tome III, Cahiers Scientifiques, fasc. 33, GauthierVillars, Paris, 1970. MR 42 \#5266.

7. P. Du Val, Homographies, quaternions and rotations, Oxford Math. Monographs, Clarendon Press, Oxford, 1964. MR 29 \#6361.

8. J. P. Fillmore, Symmetries of surfaces of constant width, J. Differential Geometry 3 (1969), 103-110. MR 40 \#858.

9. E. W. Hobson, The theory of spherical and ellipsoidal harmonics, Cambridge, 1931.

10. B. Meyer, On the symmetries of spherical harmonics, Canad. J. Math. 6 (1954), 135-157. MR 15, 525.

11. G. Pólya and B. Meyer, Sur les symétrles des fonctions sphériques de Laplace, C. R. Acad. Sci. Paris 228 (1950), 28-30. MR 10, 281.

12. F. W. Warner, Foundations of differentiable manifolds and Lie groups, Scott, Foresman, Glenview, III., 1971. MR 45 \#4312.

DEPARTAMENTO DE MATEMATICA, UNIVERSIDADE FEDERAL DE MINAS GERAIS, BELO HORIZONTE, MINAS GERAIS, BRASIL 